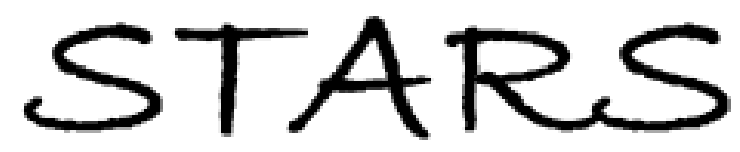

University of Central Florida

STARS

$1-1-2011$

\title{
Magnetically aligned carbon nanotube in nanopaper enabled shape-memory nanocomposite for high speed electrical actuation
}

Haibao Lu

Jihua Gou

University of Central Florida

Jinsong Leng

Shanyi Du

Find similar works at: https://stars.library.ucf.edu/facultybib2010

University of Central Florida Libraries http://library.ucf.edu

This Article is brought to you for free and open access by the Faculty Bibliography at STARS. It has been accepted for inclusion in Faculty Bibliography 2010 s by an authorized administrator of STARS. For more information, please contact STARS@ucf.edu.

\section{Recommended Citation}

Lu, Haibao; Gou, Jihua; Leng, Jinsong; and Du, Shanyi, "Magnetically aligned carbon nanotube in nanopaper enabled shape-memory nanocomposite for high speed electrical actuation" (2011). Faculty Bibliography 2010s. 1590.

https://stars.library.ucf.edu/facultybib2010/1590

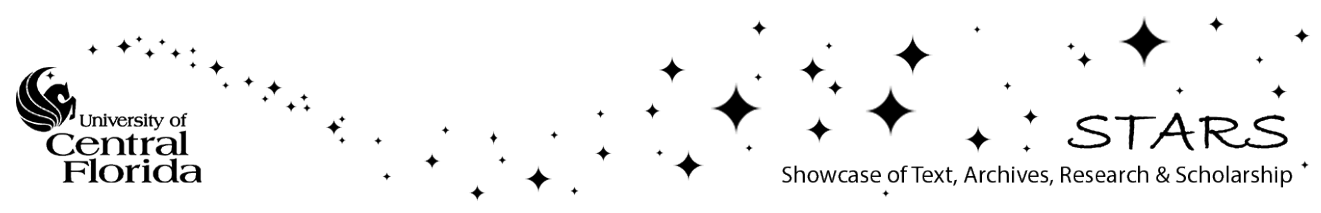




\section{Magnetically aligned carbon nanotube in nanopaper enabled shape-memory nanocomposite for high speed electrical actuation}

Cite as: Appl. Phys. Lett. 98, 174105 (2011); https://doi.org/10.1063/1.3585669

Submitted: 11 January 2011 . Accepted: 09 April 2011 . Published Online: 29 April 2011

Haibao Lu, Jihua Gou, Jinsong Leng, and Shanyi Du

\section{ARTICLES YOU MAY BE INTERESTED IN}

Synergistic effect of carbon nanofiber and carbon nanopaper on shape memory polymer composite

Applied Physics Letters 96, 084102 (2010); https://doi.org/10.1063/1.3323096

Electroactivate shape-memory polymer filled with nanocarbon particles and short carbon fibers

Applied Physics Letters 91, 144105 (2007); https://doi.org/10.1063/1.2790497

Carbon nanotube chains in a shape memory polymer/carbon black composite: To significantly reduce the electrical resistivity

Applied Physics Letters 98, 074102 (2011); https://doi.org/10.1063/1.3556621

\section{Applied Physics Reviews}

Now accepting original research 


\title{
Magnetically aligned carbon nanotube in nanopaper enabled shape- memory nanocomposite for high speed electrical actuation
}

\author{
Haibao Lu, ${ }^{1}$ Jihua Gou, ${ }^{2, a)}$ Jinsong Leng, ${ }^{1, a)}$ and Shanyi Du ${ }^{1}$ \\ ${ }^{1}$ National Key Laboratory of Science and Technology on Advanced Composites in Special Environments, \\ Harbin Institute of Technology, Harbin 150080, People's Republic of China \\ ${ }^{2}$ Department of Mechanical Engineering, University of Central Florida, Orlando 32816, USA
}

(Received 11 January 2011; accepted 9 April 2011; published online 29 April 2011)

\begin{abstract}
A new shape-memory nanocomposite that exhibits rapid electrical actuation capabilities is fabricated by incorporating self-assembly multiwalled carbon nanotube (MWCNT) nanopaper and magnetic CNTs into a styrene-based shape-memory polymer (SMP). The MWCNT nanopaper was coated on the surface to give high electrical conductivity to SMP. Electromagnetic CNTs were blended with and, vertically aligned into the SMP resin upon a magnetic field, to facilitate the heat transfer from the nanopaper to the underlying SMP. This not only significantly enhances heat transfer but also gives high speed electrical actuation. () 2011 American Institute of Physics. [doi:10.1063/1.3585669]
\end{abstract}

To date, much attention has been directed to a variety of functional materials including alloys, ceramics and polymers, which have all been found to exhibit shape memory behavior. ${ }^{1}$ Shape-memory polymers (SMPs) are an exciting class of materials that have the ability to store a temporary shape, and then recover a predetermined permanent shape when subjected to an environmental stimulus. ${ }^{2}$ As compared with other available shape-memory alloys (SMAs) at present, SMPs have many advantages, namely, wider range of shape recovery temperature, better processing ability, biocompatibility, etc. ${ }^{3}$ With these unique characteristics, SMPs currently cover a broad application area ranging from selfdeployable structure to medical implants for minimally invasive surgery. ${ }^{4}$

Shape memory behavior of SMP is usually a thermal induced process, which can be fixed and maintained until shape recovery is triggered through an external heating. Depending on the material used, stimuli can also be different. Fundamental shape-memory research is focusing on the implementation of stimuli other than direct heating to actuate SMPs, or to actuate them remotely, such as infrared light, ${ }^{5}$ water/solvent, ${ }^{6,7}$ or magnetic field. ${ }^{8}$

The demand to identify alternative strategies in order to heat SMPs above their transition temperature directed research to electrical sensitive SMPs filled with carbon nanotubes (CNTs), ${ }^{9}$ carbon black, ${ }^{10}$ conductive hybrid fibers, ${ }^{11}$ continuous conductive carbon fibers, ${ }^{12}$ conductive nanopaper ${ }^{13}$ and combination of nanopaper, randomly dispersed particles, ${ }^{14}$ etc. Electrically induced actuation allows the convenient triggering of shape recovery by passing an electrical current through a composite. However, either the examples reported to date the conductivity is still relatively low, due to the limited efficiency of discrete fillers to form percolating conductive networks. Or the random dispersed conductive fillers slightly depressed the recovery behavior of SMP. ${ }^{14}$ Here, we report the fabrication of a new shapememory nanocomposite that exhibits rapid electrical actuation capabilities is fabricated by incorporating self-assembly

\footnotetext{
${ }^{\text {a) }}$ Authors to whom correspondence should be addressed. Electronic addresses: lengjs@hit.edu.cn and Jihua.Gou@ucf.edu.
}

multiwalled CNT (MWCNT) nanopaper and magnetically aligned CNT into a styrene-based SMP. The MWCNT nanopaper was coated on the surface to give high electrical conductivity to SMP nanocomposite. CNTs were blended with and, vertically aligned into the SMP resin upon a magnetic field, to facilitate the heat transfer from the nanopaper to the underlying SMP. This not only significantly enhances heat transfer but also gives high speed electrical actuation.

Before being able to manufacture the SMP nanocomposite, fabrication of MWCNT nanopaper was first needed. The MWCNTs (L-MWNT-1020) were received in powder from Shenzhen Nanometer Gang Co., Ltd, China. The nanotube has an out diameter of 10 to $20 \mathrm{~nm}$ and $1-15 \mu \mathrm{m}$ in length. Each division of $0.6 \mathrm{~g}$ of MWCNT was added to $600 \mathrm{ml}$ of distilled water. Twenty drops of Triton X-100 nonionic surfactant were added to this mixture to ensure proper dispersion of MWCNT within the distilled water. After mechanical stirring for approximately $90 \mathrm{~s}$, the suspension was then situated in a Misonix S-4000 Sonicator and underwent two cycles of sonication at $60 \%$ amplitude for $15 \mathrm{~min}$ per cycle. That is, for every $0.6 \mathrm{~g}$ of MWCNT mixture, $30 \mathrm{~min}$ of sonication was necessary to establish quality dispersion of the MWCNTs. After the sonication process was complete, the suspension of MWCNTs and distilled water was run through a filtering system, aided by a high air pressure driver at approximately 80 psi. At this point, the newly fabricated MWCNT nanopaper was placed on a metal block. This assembly was then placed in a heating oven for $2 \mathrm{~h}$ at $120{ }^{\circ} \mathrm{C}$ to rid of any excess water.

The nickel coated CNTs are supplied from Chengdu Organic Chemicals Co. Ltd., China. These products have a diameter of 8 to $15 \mathrm{~nm}, 50 \mu \mathrm{m}$ in length. In this study, the magnetic CNTs were dispersed into the distilled water. An amount of drops of Triton X-100 surfactant were added to this mixture to ensure proper dispersion of magnetic CNTs within the distilled water. The suspension was then situated in a Misonix S-4000 Sonicator at 60\% amplitude for $15 \mathrm{~min}$. This treatment helped the as-received magnetic CNTs to reach their nanosize.

The SMP (Veriflex®S VF 62, supplied from Cornerstone Research Group, Inc., Dayton, Ohio, USA) is a 

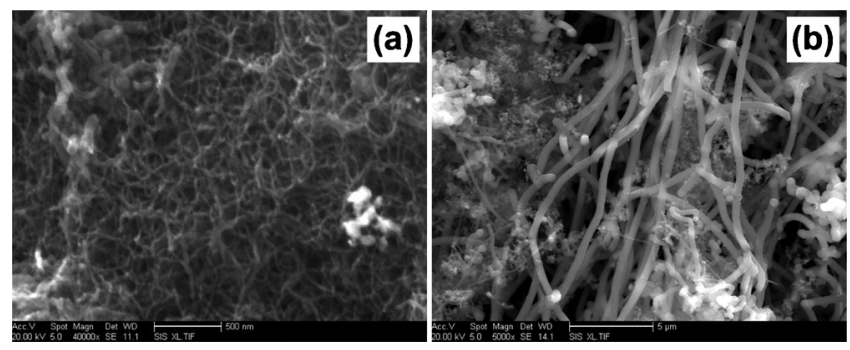

FIG. 1. (a) Morphology and structure of MWCNT nanopaper. (b) Typical surface view of the raw MWCNT arrays at magnifications of $\times 5000$ times.

styrene-based matrix. It was polymerized with the dibenzoyl peroxide harder at a fixed weight ratio of 24:1. The as treated magnetic CNTs were blended into the SMP mixture with different weight fraction of $2 \mathrm{wt} \%, 4 \mathrm{wt} \%, 6 \mathrm{wt} \%$, and 8 wt $\%$, respectively. Two magnets were placed in a way for magnetic CNTs being aligned. Simultaneously, another group of SMP nanocomposites was prepared in the same way but without applying the magnetic field.

The morphology and structure of the MWCNT nanopaper and aligned CNTs were characterized using scanning electron microscopy (SEM, MX2600FE, Camscan Co., U.K.). Figure 1(a) is a typical surface view of the raw MWCNT arrays at an accelerating voltage of $20.00 \mathrm{keV}$ and magnifications of $\times 40000$ times. The nanopaper had a porous structure with MWCNTs entangled with each other. The network structure of the nanopaper was formed by molecular interaction and mechanical inter-locking among individual nanotubes. Such a continuous network could provide conductive path for electrons, making the nanopaper electrically conductive. Figures 1(b) reveals magnetic CNTs start to align in presence of a magnetic field, and the morphology was observed at an accelerating voltage of $20.00 \mathrm{keV}$ and magnifications of $\times 5000$ times. An amount of water containing magnetic CNTs was allowed to dry. If a magnetic field was applied to the solution as it dried, the CNTs were aligned along the direction of the magnetic field.

The electrical resistivity of the SMP nanocomposite was measured with a four-terminal sensing method. The electrical resistivities of the SMP nanocomposites blending with various weight fractions of CNTs were plotted against different locations, as shown in Fig. 2. All the SMP nanocomposites were coated with one layer of nanopaper containing $1.8 \mathrm{~g}$ MWCNTs. As the weight of magnetic CNTs blended into the SMP resin increased from 0 to $8 \mathrm{wt} \%$, the average electrical resistivity of nanocomposite sample decreased from 2.076 to $0.926 \Omega \mathrm{cm}$. The more magnetic CNTs were blended into the SMP resin, the more conductive paths were formed in the SMP nanocomposite. Therefore, the electrical current amplitude and current-carrying capability increase as more electrons were forced to pass through the cross section of the bulk.

SMP nanocomposite with 8 wt $\%$ vertically aligned CNTs was further utilized to demonstrate the electrically triggered shape memory/actuation behavior. The electrical conductivity of SMP/nanopaper/CNT (vertically aligned) was $1.080 \mathrm{~S} \mathrm{~cm}^{-1}$. This high electrical conductivity allowed fast activation of shape recovery by applying a constant dc voltage. Experimentally, the recovery process was characterized using a modified bending test method, with the recovery ratio calculated based on the change in deformation angle as
CNTs are aligned in SMP resin by a magnetic field

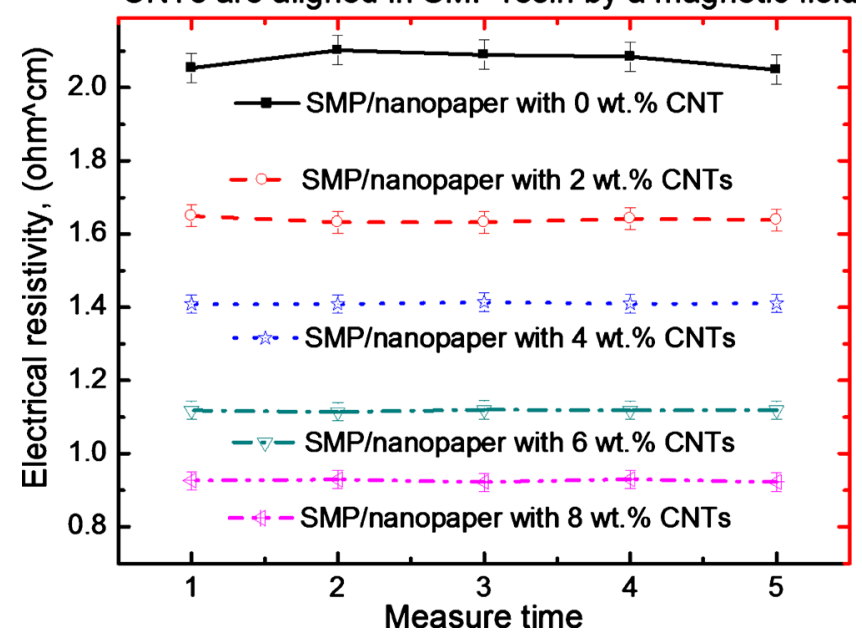

FIG. 2. (Color online) Electrical resistivity of SMP nanocomposites with MWCNT nanopaper as function of contents of vertically aligned CNTs.

function of time/temperature. ${ }^{12,15} \mathrm{~A}$ " $\Pi$ " shaped geometry was used to minimize the mechanical constraint imposed by the electrodes. The flat (permanent shape) nanocomposite specimen was bent as "U"-like shape (temporary shape) at $110{ }^{\circ} \mathrm{C}$. This temporary shape was kept until the specimen was cooled down to room temperature. No apparent recovery was seen after the deformed specimen was kept in air for $2 \mathrm{~h}$. Figure 3 shows the recovery of SMP nanocomposite specimen blended with 8 wt $\%$ vertically aligned CNTs from a fixed bent shape to its straight permanent shape under a constant dc voltage of $36 \mathrm{~V}$. The deformed nanocomposite specimen started to return to its original shape when a constant electrical current was applied for $10 \mathrm{~s}$. The specimen showed very little recovery ratio during the first $20 \mathrm{~s}$. It then started to exhibit a faster shape recovery behavior until $60 \mathrm{~s}$. During the last $15 \mathrm{~s}$, a slight change in shape recovery was observed. Finally, the nanocomposite specimen showed a 100\% recovery ratio. This fact can be attributed to the vertically aligned CNTs. These vertically aligned CNTs uniformly transferred the electrically resistive heating from nanopaper to the underlying SMP part along the vertical direction, resulting in heat transfer and dispersion significantly improved. However, they had no negative effect on the recovery ratio of SMP nanocomposite.

Meanwhile, each image was then analyzed to obtain the deformation angle, where the deformation angle shown in

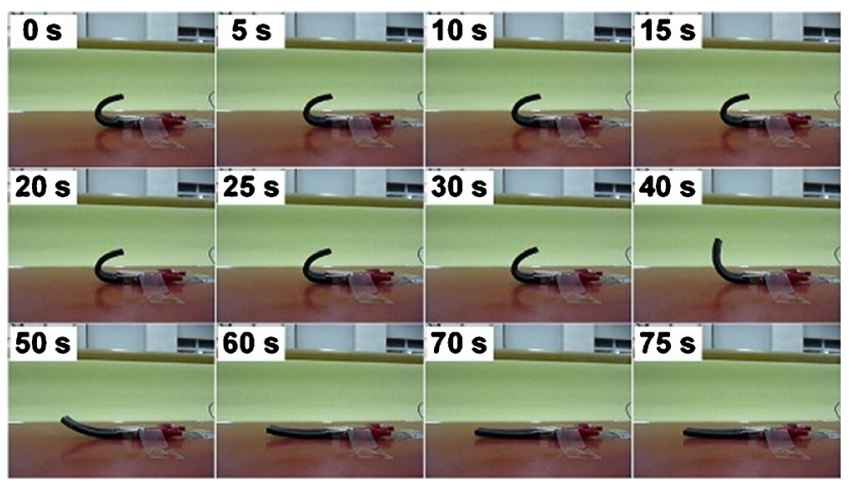

FIG. 3. (Color online) Electroactive recovery of SMP nanocomposite with 8 wt \% vertically aligned CNTs under a $36 \mathrm{~V}$ voltage. 

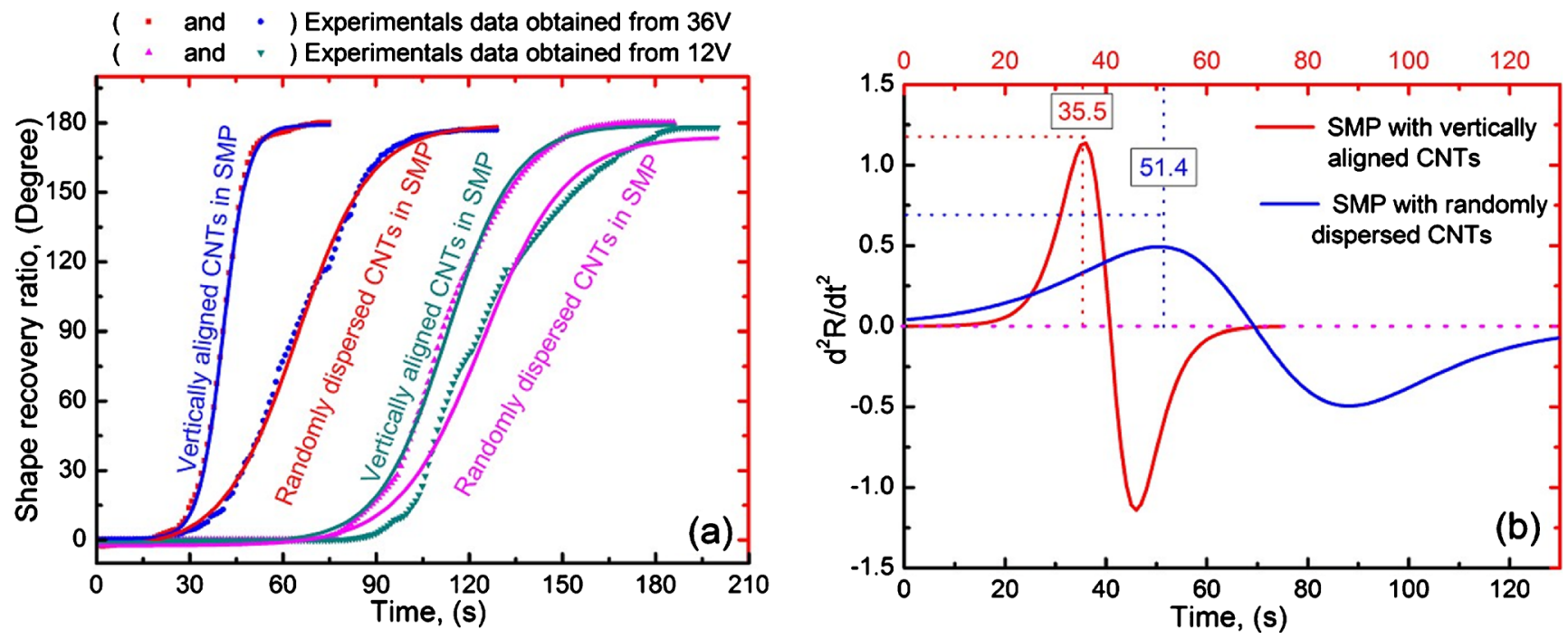

FIG. 4. (Color online) (a) shows the recovery profiles of SMP nanocomposites with, respectively, aligned and randomly dispersed 8 wt $\%$ magnetic CNTs under $12 \mathrm{~V}$ and $36 \mathrm{~V}$ voltage, respectively. (b) plots the induction and recovery times of SMP nanocomposites for the two voltages studied.

the first image is considered as 0 . Therefore, the recovery ratio is defined as, ${ }^{15}$

$$
R(\%)=\frac{\theta_{i}-\theta(t)}{\theta_{i}-\theta_{e}},
$$

where $\theta_{i}, \theta_{e}$ are the initial deformation angle of the fixed sample, the deformation angle at a given time $t$, and the deformation angle at the equilibrium state, respectively. The recovery experiments were conducted under two different dc voltages, for SMP nanocomposites with $8 \mathrm{wt} \%$ vertically aligned CNTs and randomly dispersed CNTs, respectively. The resulting recovery profiles were shown in Fig. 4(a). These curves revealed that the SMP nanocomposite with vertically aligned CNTs had the faster response behavior than that with randomly dispersed CNTs, against both two voltages. Obviously, the reduce in recovery time was the result of aligned CNTs. This experimental result revealed that the vertically aligned CNTs facilitate the heat transfer from the nanopaper to the underlying SMP, and give the SMP high speed electrical actuation.

To reveal more detailed kinetics information, the recovery data were further analyzed using a standard Boltzmann function:

$$
R(t)=A_{2}+\frac{A_{1}-A_{2}}{1+e^{\left(t-t_{0}\right) / \tau}},
$$

where $A_{1}, A_{2}, t_{0}$, and $\tau$ are the four fitting parameters. All the four fitting parameters can be obtained from the experimental results. So, the data set for each curve is " $A_{1}$ $=0.56532, A_{2}=179.05662, t_{0}=40.78832$ and $\tau=3.87918$," " $A_{1}=-4.77801, \quad A_{2}=178.67036, t_{0}=64.15814$, and $\tau$ $=12.14904$ " " $A_{1}=-4.49847, \quad A_{2}=194.38893, \quad t_{0}$ $=128.18474$, and $\tau=22.29209$ " and " $A_{1}=-0.68703, A_{2}$ $=194.92821, t_{0}=179.34018$, and $\tau=22.38264$," respectively. The fit curves had respective $R^{2}$ values of 0.99973 , $0.99731,0.99756$, and 0.99927 , and were shown as the solid lines. The second derivatives of the fit curves were then calculated and plotted in Fig. 4(b). All the second derivative plots showed a similar pattern consisting of two opposite peaks with the same height. The induction time was defined as the time between " 0 " and the first (positive) peak on the second derivative plot. This corresponds to an induction period of the recovery profile, during which the nanocomposite specimens were heated from environmental temperature to their switching temperature. It is found that the induction time of SMP nanocomposite with vertically aligned CNTs was $15.9 \mathrm{~s}$ shorter than that with randomly dispersed CNTs under the $36 \mathrm{~V}$ triggering voltage.

A series of experiments were conduct to study the synergistic effect of MWCNT nanopaper and vertical aligned CNTs on the SMP nanocomposites. The actuation of the SMP nanocomposites was achieved by electrically resistive heating of nanopaper. Electromagnetic CNTs were blended with and, vertically aligned into SMP resin upon a magnetic field, to facilitate the heat transfer from the nanopaper to the underlying SMP. This not only improves electrical conductivity and heat transfer but also gives high speed electrical actuation.

${ }^{1}$ A. Lendlein and S. Kelch, Angew. Chem., Int. Ed. 41, 2034 (2002).

${ }^{2}$ P. T. Mather, X. F. Luo, and I. A. Rousseau, Annu. Rev. Mater. Res. 39, 445 (2009).

${ }^{3}$ J. Van Humbeeck, Adv. Eng. Mater. 3, 837 (2001).

${ }^{4}$ J. S. Leng, H. B. Lu, W. M. Huang, and S. Y. Du, MRS Bull. 34, 848 (2009).

${ }^{5}$ J. S. Leng, X. L. Wu, and Y. J. Liu, J. Appl. Polym. Sci. 114, 2455 (2009).

${ }^{6}$ B. Yang, W. M. Huang, C. Li, and J. H. Chor, Eur. Polym. J. 41, 1123 (2005).

${ }^{7}$ J. S. Leng, H. B. Lv, Y. J. Liu, and S. Y. Du, Appl. Phys. Lett. 92, 206105 (2008).

${ }^{8}$ J. S. Leng, W. M. Huang, X. Lan, and S. Y. Du, Appl. Phys. Lett. 92, 204101 (2008).

${ }^{9}$ Y. J. Liu, H. B. Lv, J. S. Leng, and S. Y. Du, Compos. Sci. Technol. 69, 2064 (2009)

${ }^{10}$ H. B. Lu, K. Yu, Y. J. Liu, and J. S. Leng, Smart Mater. Struct. 19, 065014 (2010).

${ }^{11}$ J. S. Leng, H. B. Lv, Y. J. Liu, and S. Y. Du, Appl. Phys. Lett. 91, 144105 (2007).

${ }^{12}$ X. Lan, Y. J. Liu, J. S. Leng, and S. Y. Du, Smart Mater. Struct. 18, 024002 (2009).

${ }^{13}$ H. B. Lu, J. Gou, J. S. Leng, and S. Y. Du, Smart Mater. Struct. 19, 075021 (2010).

${ }^{14}$ H. B. Lu, J. Gou, J. S. Leng, and S. Y. Du, Appl. Phys. Lett. 96, 084102 (2010).

${ }^{15}$ X. F. Luo and P. T. Mather, Soft Matter 6, 2146 (2010). 